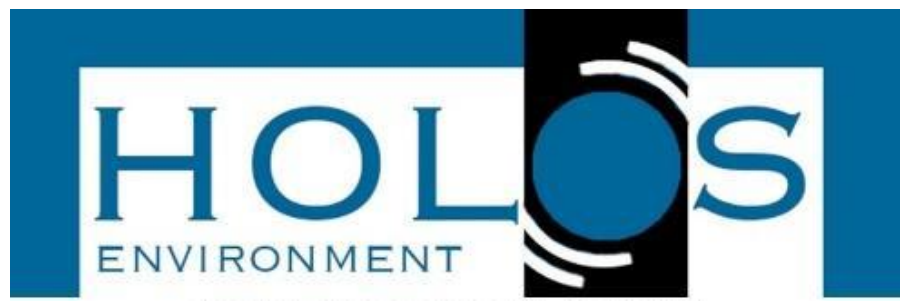

\title{
INFLUÊNCIA DO CONHECIMENTO POPULAR DE PLANTAS MEDICINAIS E A PRÁTICA DE ATIVIDADE FÍSICA NA MELHORA DA QUALIDADE DE VIDA
}

\section{THE MEDICINAL PLANTS KNOWLEDGE AND THE PHYSICAL ACTIVITY LEVEL BOTH IMPROVED THE LIFE'S QUALITY}

\author{
Lara Cavalari Santello; Maria Aparecida Marin Morales'; Mariana Freschi Bombini ${ }^{1}$
}

Artigo recebido em: 17/12/2020 e aceito para publicação em: 03/02/2021.

DOI: http://doi.org/10.14295/holos.v21i2.12434

\begin{abstract}
Resumo: O conhecimento popular de plantas medicinais e a prática da atividade física (AF) são estratégias utilizadas desde as primeiras civilizações, para uma melhor qualidade de vida. Contudo, o processo de industrialização desarticulou a transferência deste conhecimento e contribuiu para o aumento de doenças crônicas não transmissíveis (DCNT). Avaliamos o conhecimento sobre plantas medicinais e o nível de AF associados a hábitos alimentares. Foram desenvolvidos dois questionários, disponibilizados gratuitamente na plataforma Google e impressos, aplicados a 175 participantes, com média de idade de 30,74 $\pm 12,7$ (intervalo entre 17 e 77 anos). O primeiro questionário investigou o conhecimento sobre plantas medicinais e alguns hábitos alimentares. O segundo foi o Questionário Internacional de AF (IPAQ), que avaliou a intensidade de AF semanal como leve, moderada ou vigorosa. Foi observado que indivíduos mais velhos possuíam um maior conhecimento sobre as plantas medicinais $(p=0,017 ; R=0,181)$. O conhecimento sobre essas plantas também estava relacionado a uma preferência por consumo de produtos naturais $(p=0,08)$, não ingestão de refrigerante $(p=0,008)$ e a uma atividade física de rotina $(p=0,016)$. O nível de AF teve relação positiva com o conhecimento das propriedades das plantas medicinais, sendo a AF moderada a mais relacionada com esse conhecimento ( $p=0,023 ; R=0,792)$. Nossos resultados apontam uma descontinuidade na transferência desses saberes, e a necessidade de resgate de hábitos saudáveis, como a AF moderada, a ingestão de alimentos não processados e o uso de plantas medicinais para a obtenção de uma melhor qualidade de vida. Esses hábitos saudáveis podem contribuir para benefícios imediatos e de longo prazo à saúde, provavelmente por promoverem uma redução nos riscos relacionados ao aparecimento de doenças.
\end{abstract}

Palavras-chave: Alimentação saudável. Hábitos saudáveis. Produtos naturais. Redução do risco de doenças. Resgate da cultura nativa.

Abstract: The popular knowledge about medicinal plants and the physical activity (PA) practice were the therapeutic strategies use by the first civilizations for a better quality of life. However, the industrialization process disarticulated its transfer and contributed to increase chronic noncommunicable diseases (NCDs). The aim of this study was to evaluate the association between knowledge about medicinal plants and the PA level with daily feed habits. Two free online google and printed questionnaires were applied to 175 participants (mean $30.74 \pm 12.7$, interval between 17 and 77 age). The first questionnaire investigated knowledge about medicinal plants and some daily feed habits. The second was the International PA Questionnaire (IPAQ), which assesses the weekly PA intensity as mild, moderate and vigorous. We observed that older individuals had greater knowledge about medicinal plants $(p=0.017 ; R=0.181)$. Knowledge about medicinal plants was related to: preference for natural products $(p=0.08)$; no soda ingestion $(p=0.008)$ and individual physical activity $(p=0.016)$. The moderate level of PA had a positive relationship with medicinal plants knowledge $(p=$ 0.023; $R=0.792$ ). These results showed a discontinuity in transfering traditional knowledge to next

\footnotetext{
1Universidade Estadual Paulista (UNESP), Rio Claro, SP. E-mails: (lara.santello@gmail.com,
} marin.morales@unesp.br, marianafreschi@gmail.com) 
generations, indicating a need to recover healthy habits such as moderate PA practice, unprocessed food ingestion and medicinal plants use to obtain an improvement in the quality of life. These habits contribute to immediate and long-term health benefits, reducing risks related to the disease's development.

Keywords: Healthy food. Healthy habits. Natural products. Disease risks reduction. Native culture rescue.

\section{INTRODUÇÃO}

A mudança de hábitos de nômade para sedentário provocou uma verdadeira revolução no modo de vida da espécie humana. Pesquisas apontaram que o estilo de vida sedentário, decorrente da incorporação de práticas agrícolas, contribuiu para que os esqueletos se tornassem mais leves e frágeis (LARSEN, 1995; RYAN; SHAW, 2015). Essa mudança alterou, essencialmente, a habilidade de caçador para agricultor, o que foi imprescindível para o desenvolvimento e organização de uma nova sociedade humana. $\mathrm{Na}$ Grécia antiga, os jovens eram encorajados a praticar exercícios físicos para disciplinar a alma e o corpo e, assim, se prepararem para guerras (AULEY, 1994). Inúmeros relatos de povos orientais também mostram um enaltecimento à prática de exercício físico vinculado à alimentação (ARISTOTELES,1964 apud BRAGA, 2011).

A utilização de plantas na alimentação foi uma prática comum durante todo o processo de desenvolvimento social humano. Relatos da China de 3000 a.C. apontaram que o Imperador Sheng Nung consumia ginseng, e viveu por 123 anos. Algumas plantas apresentavam efeitos alucinógenos e, por isso, foram utilizadas com conotação de divindade. Estes efeitos tornaram-se uma estratégia de elevação da alma e de contato com Deus. No Egito antigo (1.500 a.C.), aromas extraídos das plantas eram utilizados em inúmeros rituais para atingir estados de elevação da alma. A mesma estratégia também foi usada na medicina oriental chinesa (2700-3000 a.C.) (MENEGUELLI et al., 2017). Um dos herbários mais antigos, o "papiros de Ebers" do Egito, tem registro de 125 plantas medicinais e 811 receitas. Na mesma região, o médico Imhotep descreveu ervas medicinais e suas curas (EBERS, 1875 apud BRUNET,1935).

No ano de 400 a.C., Hipócrates, o "Pai da Medicina", explicou em sua obra "Corpos Hipocratium", que para cada enfermidade haveria um tratamento vegetal. O livro Shen Nung's Pen Tsao descrevia a utilização de plantas aromáticas para benefícios da saúde. Anos mais tarde (60 d.C.), a obra "De Matéria Médica" descreveu a utilização de plantas aromáticas como tratamento médico. $\mathrm{O}$ autor, Pedanios Dioscorides, também teceu suas explicações sobre tais benefícios. No século XI, os mesmos princípios foram aplicados na 
prática clínica pelo médico persa Avicenna, que utilizou as propriedades medicinais de inúmeros óleos essenciais, dentre eles o óleo de rosas. A tradicional medicina ayurvédica, desenvolvida na Índia há mais de 5.000 anos, utiliza até hoje os princípios aromáticos de fitoterápicos em massagens e outras terapias. Um recente estudo reforçou a importância da alteração na capacidade olfativa como um elemento precoce de rastreio em doenças autoimunes (BOMBINI et al., 2018).

Contudo, estes conhecimentos milenares não têm sido aplicados na medicina ocidental com o mesmo afinco que o foi no passado (MOSCAVITCH et al., 2009). Na era cristã, Pelácius, médico de Nero, fez uma importante contribuição, quando catalogou mais de 500 espécies de plantas medicinais. No ano de 1484, foi impresso o primeiro livro sobre o cultivo das plantas medicinais, com base nos escritos de Dioscórides do século IV. Na Alemanha, em 1542, foram catalogadas cerca de 300 espécies de plantas medicinais em um único documento, obra que se tornou a primeira farmacopeia do mundo (ARISTOTELES, 1964 apud BRAGA, 2011). O primeiro herbário do continente americano foi feito pelo povo asteca no século XVI, e foi denominado de Manuscrito Badanius (ARISTOTELES, 1964 apud BRAGA, 2011).

A primeira farmacopeia brasileira foi minuciosamente redigida em 1929 e, atualmente, está na sua 6ำ edição (ANVISA, 2019). No Brasil, o uso das ervas com caráter curativo era frequentemente utilizado pelos índios nos rituais praticados pelos "pajés", sendo esse hábito consolidado através de gerações que também transmitiam esses conhecimentos aos seus descendentes. Esta cultura foi ainda compartilhada com os colonizadores europeus (ARISTOTELES,1964 apud BRAGA, 2011).

A prática do exercício físico como elo entre corpo e mente foi enaltecida por Platão, que reforçava a importância do exercício físico como mantenedor do equilíbrio de corpo e mente. Outro filósofo, de origem romana, chamado Juvenal, também enalteceu a importância desta relação ao dizer que: "Mens sana in corpore sano" ("Mente sã em corpo são") (HIPPOCRATE, 1983 apud GUALANO; TINUCCI, 2011). A importância da AF vinculada à alimentação obteve reconhecimento desde o início das civilizações, com a afirmação de Hipócrates: "O que é utilizado, desenvolve-se, o que não o é, desgasta-se se houver alguma deficiência de alimento e exercício, o corpo adoecerá" (HIPPOCRATES, 1983 apud GUALANO; TINUCCI, 2011).

De modo semelhante ao ocorrido na antiguidade, o início do desenvolvimento cultural da Educação Física no Brasil ocorreu, provavelmente, no período do Brasil império, com os primeiros tratados sobre a Educação Física. Em 1823, Joaquim Antônio Serpa 
elaborou o "Tratado de Educação Física e Moral dos Meninos", descrevendo que a educação incluiria a saúde do corpo e a cultura do espírito. Neste sentido, os exercícios eram divididos em categorias que priorizavam o corpo e a memória (GUTIERREZ, 1972). A prática de atividade física vinculada aos alimentos como estratégia de cura vem sendo muito comum desde tempos imemoriáveis. Por isso, os vegetais constituíram a base para o tratamento de inúmeras enfermidades (BRASIL, 2006).

Esta prática motivou estudos farmacológicos, fitoquímicos e agronômicos, que tiveram repercussão econômica, tornando este tipo de saber muito valioso. Esse conhecimento pode ser distribuído em cinco diferentes categorias (MARCONI; LAKATOS, 2003): Científico, Filosófico, Especulativo, Religioso/Teológico e Popular/Empírico.

O conhecimento popular sobre plantas medicinais fundamenta-se em experiências cotidianas, que foram transmitidas aos descendentes através dos tempos, por isso denominado de conhecimento empírico. O conhecimento popular/empírico motivou inúmeras investigações científicas (BRASILEIRO, 2008), porém, alguns destes conhecimentos foram perdidos ao longo das gerações. Para Amorozo (2002), alguns dos fatores considerados fundamentais para o enfraquecimento das práticas tradicionais de uso da fitoterapia foram: a urbanização vinculada à modernização, o êxodo rural, a mulher no mercado de trabalho, o desinteresse dos jovens pelas práticas de costumes tradicionais, a destruição de florestas e a dizimação de povos nativos.

Estes pontos têm contribuído para a diminuição ou até a não utilização de plantas medicinais, aliada à prática de AF, como primeira opção de tratamento. Diante desta situação, tornou-se fundamental conscientizar e resgatar esses conhecimentos. O presente estudo teve como objetivo investigar o conhecimento prévio dos participantes de uma pesquisa sobre o uso e a importância de plantas medicinais, aliada à prática de $\mathrm{AF}$, como estratégia de promoção de saúde e qualidade de vida. Neste estudo, foi avaliado o perfil dos indivíduos que praticam AF, o seu conhecimento sobre plantas medicinais e alguns hábitos alimentares.

\section{MATERIAL E MÉTODOS}

Nesta pesquisa, foram aplicados dois questionários com formulários online gratuitos do google e também no formato de papel para os participantes que desejassem. O primeiro questionário foi desenvolvido pelos autores desta pesquisa, para avaliar o conhecimento sobre plantas medicinais e alguns hábitos dos participantes. O questionário continha 
questões como: idade, sexo, escolaridade (opções de resposta: ensino médio incompleto, médio completo, superior incompleto e superior completo), plano de saúde (opções de resposta: sim ou não), frequência com que come "fast food" (opções de resposta: nunca, uma vez ao mês, uma vez por semana, uma ou mais vezes por semana), ingestão de refrigerante (opções de resposta: sim ou não), preferência entre suco natural e industrializado, aquisição de mais produtos naturais ou industrializados e, por último, um tópico sobre plantas medicinais, que abordava quatro aspectos: o conhecimento da planta, o conhecimento de sua utilização, se já havia utilizado e se houve melhora pelo seu uso.

As plantas avaliadas, que foram fotografadas para facilitar a identificação dos participantes da pesquisa, fazem parte das espécies mantidas pelo Jardim experimental da Unesp de Rio Claro. As plantas eleitas foram capim-santo (Cymbopogon citratus), babosa (Aloe vera), boldo (Peumus boldus), cânfora (Cinnamomun comphora), atroveran (Ochimum carnosum), arruda (Ruta graveolens), hortelanzinha (Mentha pulegium), incenso (Boswellia carterii Birdw), lavanda (Lavandula angustifolia), rubim (Leonotis nepetifolia), alecrim (Rosmarinus officinalis), menta (Mentha arvensis), canela (Cinnamomun zeylanicum), ginkobiloba (Ginkgo biloba), erva-de-santa-maria (Chenopodium ambrisioides), cavalinha (Equisetum arvense), quebra-pedra (Phyllanthus niruri), guaco (Mikania glomerata), citronela (Cymbopogon witerianus) e jurubeba (Solanum fastigiatum).

O segundo questionário aplicado foi o Questionário Internacional de AF (International Physical Activity Questionnaire - IPAQ) (MATSUDO, 2001), validado em 12 países e por 14 centros de pesquisa. O IPAQ é um questionário que identifica o tempo e a frequência de atividades físicas realizadas durante uma semana. Além disso, permite classificar a AF em moderada e vigorosa, em diferentes contextos do cotidiano, como trabalho, transporte, tarefas domésticas e lazer.

O questionário possibilita ainda a análise do tempo despendido em atividades passivas, realizadas na posição sentada. Neste estudo, utilizamos a versão curta do IPAQ, que incluiu questões que permitem identificar a frequência de atividades físicas ou exercícios físicos e ainda classificar a intensidade em moderada e vigorosa. O IPAQ permite classificar o indivíduo em quatro categorias: muito ativo, ativo, irregularmente ativo e sedentário.

O tempo despendido para responder os dois formulários foi de, até, 15 min. Todos os participantes receberam um link, para que pudessem responder as questões de forma online, e receberam um Termo de Consentimento Livre e Esclarecido (TCLE), formalizando a ciência do estudo. 


\subsection{Análise estatística}

Inicialmente, foi realizada uma análise descritiva com as características demográficas demonstradas com médias \pm desvios-padrão (DP), para as variáveis contínuas, ou por frequências e percentuais, para variáveis categóricas. A avaliação das variáveis quantitativas foi calculada pela correlação de Pearson. As análises independentes foram realizadas pelo test $T$ de Student, para verificar se existia diferenças entre as médias obtidas no estudo. Todas as análises foram realizadas no programa SPSS, versão 20.0 (Statistical Package for the Social Science), e foram consideradas significantes para valores de $p \leq 0,05$.

\section{RESULTADOS}

Inicialmente, a amostra foi composta por 178 voluntários, dos quais três não responderam a maioria das questões. Portanto, a amostra final continha 175 voluntários, sendo 109 (63\%) do sexo feminino, com média de idade de 30,74ะ12,7 (entre 17 e 77 anos). A avaliação da AF, medida pelo IPAQ, classificou os participantes em quatro categorias: muito ativo, ativo, irregularmente ativo e sedentário. As características dos voluntários estão detalhadas na tabela 1.

A análise das respostas sobre as plantas mostrou que os voluntários conheciam, em

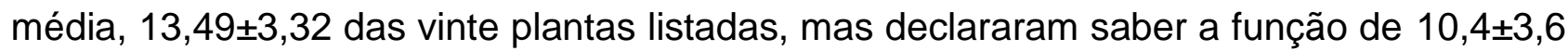
e a utilização de $8 \pm 3$, sendo que destas somente $6,8 \pm 3,1$ das plantas foram relatadas como efetivas na melhora dos sintomas. Os dados estão detalhados na tabela 2. 
Tabela 1 - Características dos voluntários.

\begin{tabular}{|c|c|}
\hline Características dos voluntários & $\mathrm{N}$ (\% válida) \\
\hline \multirow{4}{*}{ Escolaridade } & Médio incompleto $1(0,6)$ \\
\hline & Médio completo 16 (9) \\
\hline & Superior incompleto $72(40,7)$ \\
\hline & Superior completo $88(49,7)$ \\
\hline \multirow{3}{*}{ Plano de saúde } & Sim $134(77,5)$ \\
\hline & Não $39(22,5)$ \\
\hline & Nunca $55(31,8)$ \\
\hline \multirow{3}{*}{ Frequência que come fast food } & 1 vez ao mês $9(5,2)$ \\
\hline & 1 vez por semana $86(49,7)$ \\
\hline & 1 ou mais vezes por semana $23(13,3)$ \\
\hline \multirow{3}{*}{ Toma refrigerante } & Sim $102(59,3)$ \\
\hline & Não $71(40,7)$ \\
\hline & Natural $167(97,7)$ \\
\hline \multirow[t]{2}{*}{ Prefere suco natural ou industrializado } & Industrializado $1(0,6)$ \\
\hline & Ambos $3(1,8)$ \\
\hline \multirow{4}{*}{$\begin{array}{l}\text { Compra mais produtos naturais ou } \\
\text { industrializados }\end{array}$} & Natural $74(42,8)$ \\
\hline & Industrializado $81(46,8)$ \\
\hline & $\begin{array}{l}\text { Ambos } 18(10,4) \\
\text { Sim }=94(66.2)\end{array}$ \\
\hline & Não $=16(11,3)$ \\
\hline \multirow{4}{*}{$\begin{array}{l}\text { Substituiria produtos farmacológicos por } \\
\text { plantas medicinais }\end{array}$} & Sim, se houvesse mais informação $=10(7)$ \\
\hline & Depende do caso/gravidade $=19(13,4)$ \\
\hline & Como estratégia complementar $=3(2,1)$ \\
\hline & Não responderam $=36$ \\
\hline Muito Ativo & $9(5,1)$ \\
\hline Ativo & $74(42)$ \\
\hline Irregularmente ativo & 74 (42) \\
\hline Sedentário & $19(10,8)$ \\
\hline
\end{tabular}

Tabela 2 - Conhecimento sobre as vinte plantas selecionadas

\begin{tabular}{lllll}
\hline Planta envolvida & $\begin{array}{c}\text { Conhecimento } \\
\text { da planta }\end{array}$ & $\begin{array}{c}\text { Conhecimento } \\
\text { da função }\end{array}$ & Utilização & $\begin{array}{c}\text { Planta sem ação } \\
\text { comprovada }\end{array}$ \\
\hline Capim santo & $139(80,3 \%)$ & $93(53,8 \%)$ & $83(48 \%)$ & $8(9,8 \%)$ \\
Babosa & $169(97,7 \%)$ & $152(87,9 \%)$ & $116(67,1 \%)$ & $10(8,8 \%)$ \\
Boldo & $166(96 \%)$ & $159(91,9 \%)$ & $115(66,9 \%)$ & $4(3,5 \%)$ \\
Cânfora & $123(71,1 \%)$ & $97(56,4 \%)$ & $76(44,2 \%)$ & $1(1,3 \%)$ \\
Atroveram & $129(74,6 \%)$ & $61(35,5 \%)$ & $26(15,1 \%)$ & $(0 \%)$ \\
Arruda & $83(48 \%)$ & $83(48 \%)$ & $56(32,4 \%)$ & $4(7,5 \%)$ \\
Hortelanzinho & $157(91,3 \%)$ & $133(76,9 \%)$ & $135(78 \%)$ & $17(13,2 \%)$ \\
Incenso & $47(27,2 \%)$ & $30(17,4 \%)$ & $17(9,9 \%)$ & $07(43,8 \%)$ \\
Lavanda & $165(95,4 \%)$ & $109(63 \%)$ & $74(42,8 \%)$ & $12(16,4 \%)$ \\
Rubim & $15(8,7 \%)$ & $6(3,5 \%)$ & $5(2,9 \%)$ & $1(20 \%)$ \\
Alecrim & $171(98,8 \%)$ & $134(77,5 \%)$ & $130(75 \%)$ & $30(25 \%)$ \\
Menta & $160(92,5 \%)$ & $127(73,4 \%)$ & $116(67,4 \%)$ & $24(21,8 \%)$ \\
Canela & $153(89 \%)$ & $136(78,6 \%)$ & $135(78 \%)$ & $20(15,7 \%)$ \\
Ginkobiloba & $106(61,3 \%)$ & $80(46,5 \%)$ & $28(16,4 \%)$ & $3(10,7 \%)$ \\
E. sta. Maria & $71(41 \%)$ & $41(23,8 \%)$ & $26(15,2 \%)$ & $0(0 \%)$ \\
Cavalinha & $94(54,7 \%)$ & $47(27,3 \%)$ & $19(11 \%)$ & $0(0 \%)$ \\
Quebra pedra & $94(54,7 \%)$ & $79(45,7 \%)$ & $30(17,4 \%)$ & $3(10 \%)$ \\
Guaco & $110(63,6 \%)$ & $89(51,7 \%)$ & $71(41,3 \%)$ & $0(0 \%)$ \\
Citronela & $161(93,1 \%)$ & $151(87,8 \%)$ & $136(78,6 \%)$ & $3(8,2 \%)$ \\
Jurubeba & $64(37 \%)$ & $30(17,4 \%)$ & $17(10 \%)$ & $3(21,4 \%)$ \\
\hline
\end{tabular}


Foram realizadas correlações entre o conhecimento, a função, a utilização e a sensação de melhora com o uso das plantas selecionas. Estes aspectos foram correlacionados com idade, nível de AF, tempo dispendido na semana com caminhada, atividades moderadas e atividades vigorosas. Foram observadas correlações positivas, conforme detalhado na tabela 3 .

Tabela 3 - Correlação de Pearson entre as variáveis

\begin{tabular}{lcccc}
\hline $\begin{array}{c}\text { Parâmetros } \\
\text { avaliados }\end{array}$ & $\begin{array}{c}\text { Conhecimento } \\
\text { de plantas }\end{array}$ & $\begin{array}{c}\text { Conhecimento da } \\
\text { função }\end{array}$ & $\begin{array}{c}\text { Utilização da } \\
\text { planta }\end{array}$ & $\begin{array}{c}\text { Planta sem ação } \\
\text { comprovada }\end{array}$ \\
\hline Idade & $\mathrm{p}=0,017^{*}$ & $\mathrm{p}=0,017^{*}$ & $\mathrm{p}=0,001^{*}$ & $\mathrm{p}<0,001^{*}$ \\
& $\mathrm{R}=0,181$ & $\mathrm{R}=0,181$ & $\mathrm{R}=0,249$ & $\mathrm{R}=0,287$ \\
Nível de AF & $\mathrm{p}=0,288$ & $\mathrm{p}=0,008^{*}$ & $\mathrm{p}=0,157$ & $\mathrm{p}=0,095$ \\
Total caminhada & $\mathrm{R}=0,080$ & $\mathrm{R}=0,199$ & $\mathrm{R}=0,107$ & $\mathrm{R}=0,126$ \\
(tempo) & $\mathrm{p}=0,885$ & $\mathrm{p}=0,653$ & $\mathrm{p}=0,417$ & $\mathrm{p}=0,257$ \\
Total de AF & $\mathrm{R}=0,013$ & $\mathrm{R}=0,039$ & $\mathrm{R}=0,070$ & $\mathrm{R}=0,097$ \\
moderada (tempo) & $\mathrm{p}=0,388$ & $\mathrm{p}=0,398$ & $\mathrm{p}=0,023^{*}$ & $\mathrm{p}=0,676$ \\
Total de AF & $\mathrm{R}=0,075$ & $\mathrm{R}=0,073$ & $\mathrm{R}=0,792$ & $\mathrm{R}=0,036$ \\
vigorosa (tempo) & $\mathrm{p}=0,960$ & $\mathrm{p}=0,907$ & $\mathrm{p}=0,854$ & $\mathrm{p}=0,869$ \\
\hline
\end{tabular}

$\mathrm{AF}=$ Atividade Física. ${ }^{*}=$ significativo

Utilizamos o test $T$ student para as variáveis dicotômicas, referentes tanto ao perfil alimentar quanto à prática de atividade, sendo que ambas foram correlacionadas com o conhecimento de plantas medicinais. Os resultados estão detalhados na tabela 4.

Tabela 4 - Análise do perfil dos voluntários. Test T Student.

\begin{tabular}{|c|c|c|c|c|c|c|c|c|}
\hline \multirow[t]{2}{*}{ Plantas } & \multicolumn{2}{|c|}{$\begin{array}{l}\text { Ingestão de } \\
\text { refrigerante }\end{array}$} & \multicolumn{2}{|c|}{$\begin{array}{l}\text { Preferência entre } \\
\text { suco natural ou } \\
\text { industrializado }\end{array}$} & \multirow{2}{*}{\multicolumn{2}{|c|}{$\begin{array}{c}\begin{array}{c}\text { Uso de produtos } \\
\text { naturais ou } \\
\text { industrializados }\end{array} \\
\mathrm{N} \text { (Média } \pm \mathrm{DP})\end{array}$}} & \multicolumn{2}{|c|}{ Sedentarismo } \\
\hline & N (Mé & $\mathrm{ia} \pm \mathrm{DP})$ & N (Méc & $a \pm D P)$ & & & $\mathrm{N}(\mathrm{M}$ & dia \pm DP) \\
\hline Conhecer & $\begin{array}{c}\operatorname{Sim} \\
104 \\
(13 \pm 3,2)\end{array}$ & $\begin{array}{c}\text { Não } \\
71 \\
(14,3 \pm 3)^{*} \\
p=0,008\end{array}$ & $\begin{array}{c}\text { Natural } \\
170 \\
(13,5 \pm 3)\end{array}$ & $\begin{array}{c}\text { Indust. } \\
1 \\
(14 \pm 4) \\
p=0,8\end{array}$ & $\begin{array}{c}\text { Natural } \\
76 \\
(14 \pm 3,4)^{*} \\
p=0,05\end{array}$ & $\begin{array}{l}\text { Indust. } \\
81 \\
(13 \pm 3)\end{array}$ & $\begin{array}{c}\text { Sim } \\
19 \\
(12,4 \pm 2)\end{array}$ & $\begin{array}{c}\text { Não } \\
157 \\
(13,7 \pm 3)^{*} \\
p=0,016\end{array}$ \\
\hline $\begin{array}{l}\text { Conhecer } \\
\text { benefício }\end{array}$ & $\begin{array}{c}104 \\
(9,7 \pm 4,2)\end{array}$ & $\begin{array}{c}71 \\
(11,7 \pm 4)^{*} \\
p=0,00\end{array}$ & $\begin{array}{c}170 \\
(10,5 \pm 4)\end{array}$ & $\begin{array}{c}1 \\
(11 \pm 4) \\
p=0,9\end{array}$ & $\begin{array}{c}76 \\
(11,5 \pm 4,3)^{*} \\
p=0,006\end{array}$ & $\begin{array}{c}81 \\
(9,6 \pm 4)\end{array}$ & $\begin{array}{c}19 \\
(7 \pm 3,6)\end{array}$ & $\begin{array}{c}157 \\
(11 \pm 4)^{*} \\
p<0,001\end{array}$ \\
\hline Utilização & $\begin{array}{c}104 \\
(7,4 \pm 3,8)\end{array}$ & $\begin{array}{c}71 \\
(9,2 \pm 3,3)^{*} \\
p=0,001\end{array}$ & $\begin{array}{c}170 \\
(8 \pm 3,7)\end{array}$ & $\begin{array}{c}1 \\
(10 \pm 3,7) \\
p=0,6\end{array}$ & $\begin{array}{c}76 \\
(9 \pm 3,6)^{*} \\
p=0,005\end{array}$ & $\begin{array}{c}81 \\
(7,2 \pm 3,6)\end{array}$ & $\begin{array}{c}19 \\
(6,1 \pm 3)\end{array}$ & $\begin{array}{c}157 \\
(8,3 \pm 3,7)^{\star} \\
p=0,008\end{array}$ \\
\hline $\begin{array}{l}\text { Sentir } \\
\text { melhora }\end{array}$ & $\begin{array}{c}104 \\
(6,2 \pm 4)\end{array}$ & $\begin{array}{c}71 \\
(8 \pm 3,6)^{*} \\
p=0,002\end{array}$ & $\begin{array}{c}170 \\
(6,9 \pm 4)\end{array}$ & $\begin{array}{c}1 \\
(10 \pm 4) \\
\mathrm{p}=0,4 \\
\end{array}$ & $\begin{array}{c}76 \\
(7,8 \pm 4)^{*} \\
\mathrm{p}=0,007\end{array}$ & $\begin{array}{c}81 \\
(6 \pm 3,6)\end{array}$ & $\begin{array}{c}19 \\
(5 \pm 2,7)\end{array}$ & $\begin{array}{c}157 \\
(7 \pm 2,7)^{*} \\
\mathrm{p}=0,004\end{array}$ \\
\hline
\end{tabular}




\section{DISCUSSÃO}

Este é um estudo inédito sobre a relação entre o conhecimento popular de plantas medicinais e o nível de atividade física, vinculados a hábitos alimentares, que favoreceram melhores condições na qualidade de vida. Verificamos que dentre os 175 participantes (média de 30,74 $\pm 12,7$ de idade, intervalo entre 17 e 77 anos) 90,4\% tinham nível superior de educação e 77,5\% declararam ter plano de saúde. A maioria dos indivíduos amostrados afirmou ter conhecimento sobre as plantas medicinais selecionadas.

Observamos que somente $10,8 \%$ de nossa amostra era sedentária, índices inferiores aos registrados para a população brasileira (23\%) (WHO, 2018). Ao longo da história, os hábitos alimentares incorporaram conceitos culturais, sociais, filosóficos e regionais, configurando uma maneira de agregar costumes (PINHEIRO, 2008).

Neste estudo, observamos que, embora os participantes tenham mostrado uma forte preferência para consumir produtos naturais, o maior consumo foi de produtos industrializados. O fator determinante nesta escolha foi o hábito de comprar produtos industrializados, apesar da preferência registrada.

A maioria dos jovens de nossa amostra, que preferiram tomar refrigerante a suco natural, desconheciam grande parte das plantas medicinais amostradas, sendo este dado um indicativo de dietas contemporâneas e obesogênicas. A obesidade tem se mostrado um grave problema no país.

Recentemente, o Ministério da Saúde enfatizou que metade dos brasileiros estão acima do peso e $20 \%$ dos adultos são considerados obesos (BRASIL, 2020). Diante deste perfil, é possível inferir que a alimentação da população está mais associada ao consumo de alimentos industrializados do que a produtos naturais. Essa escolha, possivelmente se deve à diminuição do tempo de preparo e também ao menor tempo para consumir os alimentos, o que acaba contribuindo para escolhas rápidas, como as oferecidas em fast food ou alimentos processados, como observamos no presente estudo.

Neste sentido, a modernização e a industrialização provocaram um aumento na produção de alimentos, introduzindo na dieta opções hipercalóricas, com altos índices de açúcar e gordura, que vêm contribuindo para o aumento de doenças crônicas não transmissíveis (DCNT), como obesidade, doenças hepáticas e cardiovasculares, entre outras (MENDONÇA; ANJOS, 2004), que podem ser agravadas com a diminuição da prática de AF. As DCNT, segundo estimativas da Organização Mundial da Saúde (OMS), são responsáveis por $70 \%$ das mortes no mundo (WHO, 2014). No Brasil, as mortes 
causadas por DCNTs ultrapassam as taxas de mortalidade por doenças infecciosas e parasitárias, representando um problema econômico sério para o Sistema Único de Saúde (SUS) (BRASIL, 2008).

Contudo, felizmente, algumas pesquisas indicaram uma melhora nos hábitos alimentares, incluindo uma diminuição de $50 \%$ no consumo de refrigerantes e sucos artificiais; um aumento de $20 \%$ em AF entre os anos de 2009 a 2017; e um aumento de 5\% no consumo de frutas e hortaliças, com preferência para alimentos orgânicos, entre os anos de 2008 e 2017 (FERREIRA; COELHO, 2017).

Nesse contexto, o movimento social e econômico Slow Food tem como princípio a produção de alimentos de qualidade, que permitam o acesso justo aos consumidores (MAKUTA, 2018). Pesquisas têm demonstrado que o efeito da AF, como adjuvante do hábito alimentar, aumenta a sensação de saciedade, diminui a fome e o apetite. Evidências indicaram que exercícios físicos de alta performance suprimem a fome e podem estar relacionados com a perda de gordura corporal e aumento de grelina plasmática (DATTILO et al., 2009). Em nosso estudo, observamos que o nível de AF está diretamente correlacionado com o conhecimento das características funcionais de plantas medicinais, ao passo que AF moderada se correlaciona estreitamente com a utilização de plantas medicinas.

Existem diferenças conceituais entre os termos "atividade física" e "exercício físico". Define-se como AF qualquer movimento corporal realizado pela ação muscular esquelética, que produza movimento e gasto de energia. O exercício físico pode ser definido como uma AF planejada, estruturada e repetitiva (CASPERSEN et al., 1985). Além disso, a AF apresenta um perfil anti-inflamatório (SHARIF et al., 2018), o que, possivelmente, justifica nosso resultado, pois as pessoas que apresentaram hábitos frequentes para AF declararam não utilizar plantas medicinais.

Por outro lado, o exercício físico de alta performance, sem período de descanso adequado, pode induzir a uma desregulação do sistema imunológico, provocando uma maior suscetibilidade às doenças, o que justifica a procura por estratégias anti-inflamatórias (CERQUEIRA et al., 2020), como a utilização de plantas medicinais. Os fitoquímicos, substâncias presentes nas plantas medicinais, possuem propriedades terapêuticas que podem atuar como anti-inflamatórios e antioxidantes, que contribuem para a recuperação do pós-exercício e a melhora no desempenho esportivo do atleta (BRUNING et al., 2012).

A ingestão de alimentos e o gasto de energia são controlados por sistemas neurais complexos localizados, principalmente, na região do hipotálamo, sendo uma circuitaria 
redundante que envolve milhares de genes que reforçam a importância da escolha adequada de nutrientes e o balanço energético (LENARD; BERTHOUDH, 2009). Os nossos resultados indicam que a prática de AF estimulou escolhas alimentares mais saudáveis, aliadas a estratégias alternativas, como o uso de plantas medicinais.

A utilização do conhecimento popular sobre plantas medicinais foi fortemente difundida até meados do século $\mathrm{XX}$, mas teve um significativo declínio, devido à intensificação do uso de fármacos industrializados (BRUNING et al., 2012), demérito do saber popular sobre as plantas medicinais (AMOROZO, 2002), proibição da sua prescrição pela população leiga e até mesmo pelos médicos (CHEVALLIER, 1996).

Estas constatações anteriores corroboram com os resultados desse estudo, pois verificamos que os indivíduos mais velhos apresentavam hábitos mais saudáveis e com correlação positiva ao conhecimento sobre plantas medicinais. De modo intrigante, os jovens, que supostamente teriam acesso à informação mais fácil e rápida, não conheciam a maioria das espécies das plantas listadas, denotando um conhecimento superficial desta faixa etária sobre plantas medicinais.

Embora tivessem ouvido falar das plantas, desconheciam as suas funções, dados estes que reforçam a descontinuidade na transferência do saber sobre plantas para as gerações seguintes, possivelmente devido ao desinteresse estimulado por condições econômicas e culturais externa citadas por Amorozo (2002).

O desenvolvimento e a ascensão do conhecimento químico, que ocorreu após a segunda metade do século XX (época da consolidação da indústria farmacêutica), colaborou para que novas substâncias fossem isoladas e sintetizadas em laboratório, promovendo uma substituição exacerbada da fitoterapia pelos medicamentos sintéticos (CECHINEL E YUNESR, 1998). Outro possível evento que pode ter contribuído para a desarticulação do uso medicinal de plantas foi a mudança de hábitos rurais para urbanoindustriais, que influenciaram mudanças comportamentais, econômicas e culturais, como a medicina moderna (AMOROZO, 2002).

Situação como essa foi comprovada em nossa pesquisa, uma vez que a maioria dos entrevistados que conheciam as plantas foram os voluntários de idade mais avançada. Pode-se dizer que a população mais velha teve um contato maior com a grande variedade de plantas medicinais, quando comparados com as gerações mais novas. Contudo, outros trabalhos realizados na região nordeste do Brasil indicaram que o uso de plantas medicinais foi a primeira medida de tratamento escolhida pela população (PINTO; AMOROZO, 2006; TEIXEIRA et al., 2014), assim como observado também na região de Minas Gerais 
(ARNOUS et al., 2005). Estes dados foram atribuídos ao baixo custo do produto e ao conhecimento prévio advindo de gerações anteriores. Estes trabalhos fortaleceram as práticas tradicionais do uso de plantas medicinais e contribuíram para a valorização da flora local (PINTO; AMOROZO, 2006; TEIXEIRA et al., 2014; ARNOUS et al., 2005). No passado, as plantas medicinais eram o principal meio terapêutico para o tratamento da população (ACCORSI, 2000), caracterizando-se como uma alternativa viável para o tratamento de inúmeras doenças.

Esta alternativa deve ser mais incentivada e explorada no Brasil, uma vez que o país possui a maior diversidade botânica do planeta (cerca de 200.000 espécies catalogadas) (LEWINSOHNT; PRADO, 2002). Os nossos dados permitem inferir que o conhecimento sobre a utilização de plantas está vinculado à melhora na qualidade de vida, visto que a maioria dos indivíduos que faziam uso dessa prática eram mais velhos e também praticavam, diariamente, $\mathrm{AF}$, além de ter um menor consumo de alimentos processados.

Em consonância com a nossa percepção da importância de conhecimentos empíricos/tradicionais, a OMS tem recomendado a inclusão de práticas da medicina caseira, adotadas antigamente, como estratégia terapêutica para a saúde pública (OMS, 1979). Diante do exposto, sabendo dos efeitos da industrialização sobre o estilo de vida, incluindo o sedentarismo, a diminuição da valorização dos conhecimentos populares sobre plantas medicinais e um consequente aumento das DCNTs, que causaram $72 \%$ das mortes e $75 \%$ dos gastos com atenção à saúde no SUS, fica explícita a necessidade de resgatar e valorizar o conhecimento da medicina popular, bem como de alguns hábitos tradicionais que possibilitam a diminuição dos gastos com a saúde e garantem a prevenção de muitas doenças. A necessidade de estimular o conhecimento sobre as plantas medicinais também foi reconhecida pelo governo, principalmente por favorecer a população de baixa renda, 0 aumento de consumo de produtos naturais e a eficácia no tratamento de enfermidades (FOLHA DE SÃO PAULO, 2015).

O Governo Federal brasileiro instituiu a Política Nacional de Plantas Medicinais e Fitoterápicos (PNPMF) com o Decreto Presidencial 5.813 de 22/6/2006 (BRASIL, 2006), destinado à promoção da saúde e qualidade de vida da população. Além da PNPMF, o SUS implementou também 29 práticas integrativas e complementares à saúde pública, que incluem a Medicina Tradicional Chinesa (Acupuntura); Homeopatia; Fitoterapia; Medicina Antroposófica; Termalismo (Crenoterapia) (BRASIL, 2006); Arteterapia; Ayurveda; Biodança; Dança Circular; Meditação; Musicoterapia; Naturopatia; Osteopatia; Quiropraxia; Reflexoterapia; Reiki; Shantala; Terapia Comunitária Integrativa; Yoga (BRASIL, 2017); 
apiterapia; aromaterapia; bioenergética; constelação familiar; cromoterapia; geoterapia; hipnoterapia; imposição de mãos; ozonioterapia e terapia de florais (BRASIL, 2018).

O reconhecimento do governo sobre a utilização de práticas alternativas reforça a importância de conservar o saber popular, ainda que a aplicação efetiva seja discreta. Reiteramos que a inserção de novos elementos culturais, principalmente os advindos do processo de industrialização, desarticulou a transmissão do conhecimento popular, ameaçando, assim, um patrimônio cultural de valor inestimável para as futuras gerações.

\section{CONCLUSÃO}

O resgate e a consciência de que hábitos saudáveis vinculados ao incentivo de práticas de $\mathrm{AF}$, o consumo de alimentos não processados e a utilização de plantas medicinais podem contribuir para uma melhor qualidade de vida do cidadão e, juntos, podem diminuir a incidência de inúmeras doenças.

\section{AGRADECIMENTOS}

Ao departamento de Biologia Geral e Aplicada do IB/Unesp de Rio Claro-SP pelo apoio, ajuda técnica e incentivo ao desenvolvimento da pesquisa. Ao Jardim experimental da Unesp de Rio Claro, que contém os exemplares das plantas selecionadas, e ao jardineiro João Cidnei Covre pelo apoio técnico.

\section{REFERÊNCIAS}

ACCORSI, W. R. Medicina natural, um novo conceito. A fórmula: guia de negócios. Revista Espaço para a Saúde, v. 2, n. 4, p. 5-8, 2000.

AMOROZO, M.C.M. Uso e diversidade de plantas medicinais em Santo Antonio do Leverger, MT, Brasil. Acta Bot. Bras., São Paul, v.16, n. 2, p189-203, Abr. 2002.

https://doi.org/10.1590/S0102-33062002000200006

ANVISA. Agencia Nacional De Vigilância Sanitária. Resolução da diretoria colegiada- RDC no 298/2019, de 14 de agosto de 2019. Disponível em:

http://antigo.anvisa.gov.br/legislacao\#/visualizar/403911. Acesso em : 14 ago. 2019.

ARNOUSA, H.; SANTOS, A.S.; BEINNER, R.P.C. Plantas medicinais de uso caseiro conhecimento popular e interesse por cultivo. Revista Espaço para a Saúde, v. 6, n.2 , p. 1- 6, 2005. 
AULEY, D. M. A history of physical activity, health and medicine. Journal of the Royal Society of Medicine, v. 87, n.1, p. 32-35, jan. 1994.

LEWINSOHNT, M.; PRADO, P. (Ed.). Biodiversidade brasileira: síntese do estado atual do conhecimento. São Paulo: Contexto, 2002.

BOMBINI, M.F.; PERES, F.A.; LAPA, A.T.; SINICATO, N.A.; QUENTAL, B.; PINCELLI, Á.S.M.; AMARAL, T.N.; GOMES, C.C.; DEL RIO, A.P.; MARQUES-NETO, J.F.; COSTALLAT, L.T.L.; FERNANDES, P.T.; CENDES, F.; RITTNER, L.; APPENZELLER, S. Olfactory function in systemic lupus erythematosus and systemic sclerosis. A longitudinal study and review of the literature.

Autoimmunity Reviews, v. 17, n. 14, p.405-412, apr. 2018.

https://doi.org/10.1016/..autrev.2018.02.002

BRAGAC.M. Histórico da utilização de plantas medicinais. Monografia (Conclusão de Curso) Universidade de Brasília / Universidade Estadual de Goiás, 2011.

BRASIL. Ministério da Saúde. Portaria n 702 - Altera a Portaria de Consolidação no 2/GM/MS, de 28 de setembro de 2017, para incluir novas práticas na Política Nacional de Práticas Integrativas e Complementares - PNPIC. Diário Oficial, Brasília, 2018.

BRASIL. Ministério da Saúde (MS). Secretaria de Vigilância à Saúde. Secretaria de Atenção à Saúde. Diretrizes e recomendações para o cuidado integral de doenças crônicas nãotransmissíveis. Brasília, 2008.

BRASIL. Ministério da Saúde. Mais da metade dos brasileiros está acima do peso e $20 \%$ obesos, alerta Ministério da Saúde. Disponível em: https://pebmed.com.br/mais-da-metade-dosbrasileiros-esta-acima-do-peso-e-20-obesos-alerta-ministerio-da-saude/. Acesso em: 04 mar. 2020.

BRASIL. Presidência da República. Decreto № 5.813 de 22 de junho de 2006. Política Nacional de Plantas Medicinais e Fitoterápicas. Diário Oficial da União, Brasília, 23 de junho de 2006.

BRASIL. Portaria no. 971, de 03 de maio de 2006 - Política Nacional de Práticas Integrativas e Complementares (PNPIC) no Sistema Único de Saúde. Diário Oficial da União, Brasília, 04 maio 2006.

BRASIL. Ministério da Saúde. Portaria o 849, de 27 de março de 2017. Inclui a arteterapia, ayurveda, biodança, dança circular, meditação, musicoterapia, naturopatia, osteopatia, quiropraxia, reflexoterapia, reiki, shantala, terapia comunitária integrativa e yoga à política nacional de práticas integrativas e complementares. Diário Oficial da União, Brasília, 28 mar. 2017.

BRASILEIRO, B.G.; PIZZIOLO, V.R.; MATOS, D.S.; GERMANO, A.M.; JAMAL, C.M. Plantas medicinais utilizadas pela população atendida no "Programa de Saúde da Família", Governador Valadares, MG, Brasil. Revista Brasileira de Ciências Farmacêuticas, São Paulo, v. 44, n. 4, p.631-636, dez. 2008. https://doi.org/10.1590/S1516-93322008000400009

BRUNING, M.C.R.; MOSEGUIG, B.G.; VIANNA, C.M.M. A utilização da fitoterapia e de plantas medicinais em unidades básicas de saúde nos municípios de Cascavel e Foz do lguaçu - Paraná: a visão dos profissionais de saúde. Ciência \& Saúde Coletiva. v. 17, n.10, p. 2675-2685, 2012. http://dx.doi.org/10.1590/S1413-81232012001000017

CASPERSEN, C.J.; POWELL, K.E.; CHRISTENSON, G.M. Physical activity, exercise, and physical fitness: definitions and distinctions for health-related research. Public health reports. $v$. 100, n. 2, p.126-131, abr. 1985. 
CECHINEL FILHO, V.; YUNESR, A. Estratégias para a obtenção de compostos farmacologicamente ativos a partir de plantas medicinais: conceitos sobre modificação estrutural para otimização da atividade. Química Nova. São Paulo, v. 21, n.1, p. 99-105, fev. 1998. https://doi.org/10.1590/S0100-40421998000100015

CERQUEIRA, E.; MARINHO, D.A.; NEIVA, H.P.; LOURENÇO, O. Inflammatory Effects of High and Moderate Intensity Exercise-A Systematic Review. Frontiers in Physiology. v. 10, jan. 2020. https://doi.org/10.3389/fphys.2019.01550

CHEVALLIERA. The encyclopedia of medicinal plants: an excellent guide to over 500 of the more well-known medicinal herbs from around the world. London: Dorling Kindersley, 1996. 336p.

CONFERÊNCIA INTERNACIONAL SOBRE CUIDADOS PRIMÁRIOS DE SAÚDE: al-a-ata-urss, 1978: relatório final. Ministério da Saúde. Organização mundial de saúde fundo das nações unidas para infância: OMS-UNICEF. Brasília, 1979.

DATTILO, M.; MEDEIROS, R.; SAAD, M. Aspectos fisiológicos do comportamento alimentar e sua relação com o exercício físico. Revista Digital, Buenos Aires, v. 14, n. 134 , 2009. Disponível em : http://www.efdeportes.com/efd134/comportamento-alimentar-e-sua-relacao-com-o-exerciciofisico.htm. Acesso em: jul. 2019.

BRUNET, Plerre; MIELT, Aldo. Histoire des ciences. Paris: Antiquité, 1935.

FERREIRA, A.S.; COELHO, A.B. O Papel dos Preços e do Dispêndio no Consumo de Alimentos Orgânicos e Convencionais no Brasil. Rev. Econ. Sociol. Rural. v. 55, n.4, 2017. http://dx.doi.org/10.1590/1234-56781806-94790550401

FRIAS, M.C. Farmacêuticas investem r\$332 mil em fitoterápicos. Folha de São Paulo, São Paulo, 19 maio 2015. Disponível em: http://www.abifisa.org.br/noticia/23-07-2015-mercado-abertofarmaceuticas-investem-r-332-mi-em-fitoterapicos .

GUALANO, B.; TINUCCI, T. Sedentarismo, exercício físico e doenças crônicas.Rev. bras. Educ. Fís. Esporte, São Paulo, v. 25, p.37-43, Dez. 2011.

GUTIERREZ, W. História da educação física. 4.ed. Porto Alegre: IPA, 1972. 50 p.

MARCONI, M.A.; LAKATOS, E.M. Fundamentos de metodologia científica. 5. ed. São Paulo: Atlas, 2003. 310p.

LARSEN, C. S. Biological changes in human populations with agriculture. Annual Review of Anthropology, v. 24, p.185-213, out. 1995.

https://doi.org/10.1146/annurev.an.24.100195.001153

LENARD, N.R., BERTHOUD, H.R. Central and peripheral regulation of food intake and physical activity: pathways and genes. Obesity (Silver Spring). v. 3, Suppl. 3, p.11-22, dez. 2009. https://doi.org/10.1038/oby.2008.511

LORENZO, L. História da educação e da pedagogia. São Paulo: Senac Nacional, 1981. 292p.

MAKUTA, G. Biodiversidade, arca do gosto e fortalezas slow food: um guia para entender o que são, como se relacionam com o que comemos e como podemos apoiá-las.São Paulo:

Fundação Slow Food para Biodiversidade, 2018. 
MATSUDO, S.; ARAÚJO, T.; MATSUDO, V.; ANDRADE, D.; ANDRADE, E. OLIVEIRA, L.C.; BRAGGION, G. Questionário Internacional de Atividade Física (IPAQ): Estudo de validade e reprodutibilidade no Brasil. Atividade Física \& Saúde. v. 6, n. 2, p. 6-18, 2001 https://doi.org/10.12820/rbafs.v.6n2p5-18

MENDONÇA, C.P.; ANJOS, L.A. Aspectos das práticas alimentares e da AF como determinantes do crescimento do sobrepeso/obesidade no Brasil. Caderno Saúde Pública, Rio de Janeiro, v. 20, n.3, p.698-709, jun. 2004

MENEGUELLI, A.Z.; RIBEIRO, S.B.; JUNIOR, G.A.L.; SPIROTTO, E.O.; SOUZA, J.H.G. A utilização de plantas medicinais e fitoterápicas na saúde pública brasileira. Enfermagem e Saúde Coletiva, São Paulo, v. 1, n. 01, p. 2-12, 2017.

MOSCAVITCH, S.D.; SZYPER-KRAVITZ, M.; SHOENFELD, Y. Autoimmune pathology accounts for common manifestations in a wide range of neuro-psychiatric disorders: the olfactory and immune system interrelationship. Clinical immunology. v. 3, n. 130, p .235-243, 2009. https://doi.org/10.1016/i.clim.2008.10.010

PINHEIRO, K.A.P.N. História dos hábitos alimentares ocidentais. Universitas Ciências da Saúde. Brasília, v. 03, n.01, p.173-190, 2005. https://doi.org/10.5102/ucs.v3i1.553

PINTO, E.P.P.; AMOROZO M.C.M.; A Furlan. Conhecimento popular sobre plantas medicinais em comunidades rurais de mata atlântica - Itacaré, BA, Brasil._Acta botânica brasileira, São Paulo, v. 20, n.4, p.751-762, out. 2006. https://doi.org/10.1590/S0102-33062006000400001

RYAN, T.M.; SHAW, C.N. Gracility of the modern Homo sapiens skeleton is the result of decreased biomechanical loading. PNAS, v. 2, n.112, p.372-377, jan. 2015.

https://doi.org/10.1073/pnas.1418646112

SHARIF, K.; WATAD, A.; BRAGAZZI, N.L.; LICHTBROUN, M.; AMITAL, H.; YEHUDA SHOENFELD, Y. Physical activity and autoimmune diseases: get moving and manage the disease. Autoimmunity reviews, v.17, n.1, p. 53-72, jan. 2018.

https://doi.org/10.1016/i.autrev.2017.11.010

TEIXEIRA, A.H.T.; BEZERRA, M.M.; CHAVES, H.V.; VAL, D.R.; FILHO, S.M.P.; SILVA, A.A.R. Conhecimento popular sobre o uso de plantas medicinais no município de sobral-Ceará, Brasil. Sanare, v. 13, n. 1, p. 23-28, 2014. https://doi.org/10.36925/sanare.v13i1.429

WHO Global. Action plan on physical activity 2018-2030: more active people for a healthier world. Geneva: WHO, 2018. WHO, 4 jun. 2018. http://apps.who.int/iris/bitstream/handle/10665/272722/9789241514187-eng.pdf ]

WORLD HEALTH ORGANIZATION. Health statistics and information systems: estimates for 2000-2012. Geneva: WHO, 03 nov. 2014. Disponível em:

http://www.who.int/healthinfo/global burden disease/estimates/en/index1.html 